\title{
Evaluation of the potential bioaccumulation ability of the blood cockle (Anadara granosa L.) for assessment of environmental matrices of mudflats.
}

\begin{abstract}
The spatial distribution of 19 polycyclic aromatic hydrocarbons (tPAHs) was quantified in aquacultures located in intertidal mudflats of the west coast of Peninsular Malaysia in order to investigate bioaccumulation of PAH in blood cockles, Anadara granosa (A. granosa). Fifty-four samples from environmental matrices and A. granosa were collected. The sampling locations were representative of a remote area as well as PAH-polluted areas. The relationship of increased background levels of PAH to anthropogenic PAH sources in the environment and their effects on bioaccumulation levels of A. granosa are investigated in this study. The levels of PAH in the most polluted station were found to be up to ten-fold higher than in remote areas in blood cockle. These high concentrations of PAHs reflected background contamination, which originates from distant airborne and waterborne transportation of contaminated particles. The fraction and source identification of PAHs, based on fate and transport considerations, showed a mix of petrogenic and pyrogenic sources. The relative biota-sediment accumulation factors (RBSAF), relative bioaccumulation factors from filtered water (RBAFw), and from suspended particulate matter (SPM) (RBAFSP) showed higher bioaccumulations of the lower molecular weight of PAHs (LMWs) in all stations, except Kuala Juru, which showed higher bioaccumulation of the higher molecular weight of PAHs (HMWs). Calculations of bioaccumulation factors showed that blood cockle can accumulate PAHs from sediment as well as water samples, based on the physicochemical characteristics of habitat and behaviour of blood cockles. Correlations among concentrations of PAHs in water, SPM, sediment and A. granosa at the same sites were also found. Identification of PAH levels in different matrices showed that A. granosa can be used as a good biomonitor for LMW of PAHs and tPAHs in mudflats. Considering the toxicity and carcinogenicity of PAHs, the bioaccumulation by blood cockles are a potential hazard for both blood cockles and their consumers.
\end{abstract}

Keyword: PAHs; Anadara granosa; Bioaccumulation; Biomonitor; Mudflats; Malaysia. 